\title{
POLYNOMIALS AND TENSORS OF BOUNDED STRENGTH
}

\author{
ARTHUR BIK, JAN DRAISMA, AND ROB H. EGGERMONT
}

\begin{abstract}
Notions of rank abound in the literature on tensor decomposition. We prove that strength, recently introduced for homogeneous polynomials by Ananyan-Hochster in their proof of Stillman's conjecture and generalised here to other tensors, is universal among these ranks in the following sense: any non-trivial Zariski-closed condition on tensors that is functorial in the underlying vector space implies bounded strength. This generalises a theorem by Derksen-EggermontSnowden on cubic polynomials, as well as a theorem by Kazhdan-Ziegler which says that a polynomial all of whose directional derivatives have bounded strength must itself have bounded strength.
\end{abstract}

\section{INTRODUCTION AND MAIN THEOREM}

For any Zariski-closed affine cone $X$ that spans a vector space $V$, the $X$-rank of $v \in V$ is the minimal number of terms across all expressions of $v$ as a sum of vectors in $X$ [Lan12, Section 5.2.1]. If $X$ is the cone over a Veronese embedding of a projective space, then the $X$-rank is the Waring rank; if it is the cone over the Segre embedding of a product of projective spaces, then the $X$-rank is the ordinary tensor rank; and if $X$ is the (reducible) variety of $d$-way tensors that are a product of a vector with a $(d-1)$-way tensor, then the $X$-rank is the slice rank [TS16]. Each of these ranks behaves functorially in the underlying vector space(s) and is lower-bounded by the strength that we will introduce below. In particular, in each of these cases, bounded X-rank implies bounded strength.

This is not a coincidence. In this paper we establish that if the type of tensors is fixed but the underlying vector space(s) are not, then any non-trivial Zariski-closed condition that is functorial in the underlying vector space(s) implies bounded strength. Note that bounded X-rank is typically not a closed condition, but our result does apply to its closure, bounded border X-rank.

We set the stage by discussing our result in detail for homogeneous polynomials (symmetric tensors), and then treat the cases of alternating and ordinary tensors more succinctly. We have chosen this case-by-case treatment, rather than a more uniform treatment via polynomial functors, to make the paper more immediately useful for researchers in tensor decomposition. Moreover, in the symmetric and alternating cases there are conditions on the characteristic of the ground field that are not needed in the case of ordinary tensors; and finally, this case-by-case treatment allows us to give explicit case-by-case bounds.

1.1. Strength of homogeneous polynomials. Let $K$ be a perfect and infinite field, write $\mathrm{Vec}=\mathrm{Vec}_{K}$ for the category of finite-dimensional $K$-vector spaces, let $V \in \mathrm{Vec}$, and let $d \in \mathbb{Z}_{\geq 2}$. We write $S^{d} V$ for the $d$-th symmetric power of $V$, and we

The authors were supported by the NWO Vici grant entitled Stabilisation in Algebra and Geometry. 
informally refer to elements of $S^{d} V$ as homogeneous polynomials of degree $d$ in $\operatorname{dim} V$ variables.

This paper concerns decompositions of $q \in S^{d} V$ of the form

$$
q=r_{1} s_{1}+\cdots+r_{k} s_{k}
$$

where $r_{i} \in S^{e_{i}} V$ and $s_{i} \in S^{d-e_{i}} V$ for suitable natural numbers $e_{i} \in\{1, \ldots, d-1\}$. The minimal number $k$ of terms among all such decompositions of $q$ is called the strength $S(q)$ of $q$. This term was introduced in [AH16], except that we have taken the liberty of adding 1 to the strength defined there-so that, for instance, our strength is subadditive. By taking all $e_{i}$ equal to 1 , we obtain the bound $S(q) \leq \operatorname{dim} V$; but we will be interested in upper bounds that do not depend on $\operatorname{dim} V$.

1.2. Closed subsets of $S^{d}$. We equip $S^{d} V$ with the Zariski topology. A closed subset of $S^{d}$ is a rule $X$ that assigns to every $V \in$ Vec a closed subset $X(V)$ of $S^{d} V$ in such a manner that for any linear map $\varphi \in \operatorname{Hom}_{\mathrm{Vec}}(V, W)$ the $d$-th symmetric power $S^{d} \varphi$ maps $X(V)$ into $X(W)$. In particular, if we set $X_{n}:=X\left(K^{n}\right)$, then $X_{n}$ is stable under the group $\mathrm{GL}_{n}$, and the map $S^{d} \pi: S^{d} K^{n} \rightarrow S^{d} K^{n-1}$ coming from the projection $\pi: K^{n} \rightarrow K^{n-1}$ forgetting the last coordinate maps $X_{n}$ into $X_{n-1}$-indeed, onto $X_{n-1}$, as one sees using the $d$-th symmetric power of any section $K^{n-1} \rightarrow K^{n}$ of $\pi$.

\subsection{Examples.}

Example 1. Let $d=3$, fix $k \in \mathbb{Z}_{\geq 0}$, and let $Y_{3, k}(V) \subseteq S^{d} V$ be the set of all elements of strength at most some fixed number $k$, i.e., the set of cubics that can be expressed as a sum of $k$ products of a linear form and a quadric. In [DES17] it is proved that $Y_{3, k}(V)$ is Zariski-closed. Since $S^{d} \varphi$ maps a decomposition $\circledast$ into another such decomposition, $Y_{3, k}$ is a closed subset of $S^{3}$.

Example 2. For arbitrary $d$, we do not know whether the set of elements in $S^{d} V$ of strength at most $k$ is closed. Let $Y_{d, k}(V)$ be its closure, the set of elements in $S^{d} V$ of border strength at most $k$; then $Y_{d, k}$ is a closed subset of $S^{d}$. The parameterisation ( shows that the topological dimension of $Y_{d, k}(V)$ is at most a polynomial in $\operatorname{dim} V$ of degree $d-1$. As $\operatorname{dim} S^{d} V$ has degree $d$ in $\operatorname{dim} V$, for $\operatorname{dim} V \gg 0$ the closed subset $Y_{d, k}(V)$ is not equal to $S^{d} V$. This conclusion is abbreviated as $Y_{d, k} \subsetneq S^{d}$.

Note that though the set of elements in $S^{d} V$ of strength at most $k$ is not necessarily closed, we will find (by means of Theorem 4) that its closure only contains elements of strength at most $N$ for some $N \geq k$ (provided we work over a field of characteristic 0 or characteristic greater than $d$ ).

Example 3. The paper [KZ18] concerns polynomials all of whose directional derivatives have bounded strength. In our notation, this leads to the closed set $Z_{d, k} \subseteq S^{d}$ defined by

$$
Z_{d, k}(V):=\left\{q \in S^{d} V \mid \forall x \in V^{*}:\langle x, q\rangle \in Y_{d-1, k}(V)\right\},
$$

where $\langle., .\rangle:. V^{*} \times S^{d} V \rightarrow S^{d-1} V$ is the natural pairing. This set is potentially larger than the one in [KZ18] since we allow directional derivatives to have strength larger than $k$, as long as their border strength is at most $k$.

If, as in [KZ18], char $K=0$ or char $K>d$, then for every nonzero $x \in V^{*}$ the linear map $\langle x,\rangle:. S^{d} V \rightarrow S^{d-1} V$ is surjective. Hence if $Y_{d-1, k}(V) \subsetneq S^{d-1} V$, then also 
$Z_{d, k}(V) \subsetneq S^{d} V$. As the former happens for all $V$ with $\operatorname{dim} V \gg 0$, so does the latter, so $Z_{d, k} \subsetneq S^{d}$.

1.4. Main theorem for polynomials. Our main theorem is a converse to Example 2. under mild conditions, in any closed $X \subsetneq S^{d}$ the strength is uniformly bounded, independently of $\operatorname{dim} V$.

Theorem 4. Fix $d \in \mathbb{Z}_{\geq 2}$ and assume that $K$ is a perfect and infinite field with char $K=0$ or char $K>d$. Then for any closed $X \subsetneq S^{d}$ there exists an $N$ such that for all finitedimensional vector spaces $V$ the strength of all elements in $X(V)$ is at most $N$.

The theorem has a reformulation in terms of limits. Set $S_{\infty}^{d}:=\lim S^{d} K^{n}$, the inverse limit under the maps mentioned in 1.2 The notion of strength extends naturally to $S_{\infty}^{d}$, except that it can now be infinite.

Example 5. In [DES17] it is proved that the polynomial

$$
f=x_{1} y_{1} z_{1}+\cdots+x_{n} y_{n} z_{n} \in S^{3} K^{3 n},
$$

where $x_{1}, y_{1}, z_{1}, \ldots, x_{n}, y_{n}, z_{n}$ is the standard basis of $K^{3 n}$, has strength $n$, and that

$$
\sum_{i=1}^{\infty} x_{i} y_{i} z_{i} \in S_{\infty}^{3}
$$

has infinite strength.

To find higher-degree examples of polynomials with high strength, we have the following variant of an argument used in [DES17].

Lemma 6. Suppose that $q \in S^{d} V$ has $S(q) \leq k$. Then for some $\ell \leq k$ there exists an $\ell$ dimensional subspace $W \subseteq V$ such that the image q of $q$ in $S^{d}(V / W)$ has $S(\langle x, \tilde{q}\rangle) \leq 2(k-\ell)$ for all $x \in(V / W)^{*}$.

Proof. Arrange a decomposition $q=r_{1} s_{1}+\cdots+r_{k} s_{k}$ such that $r_{1}, \ldots, r_{\ell}$ are linear and $r_{j}, s_{j}$ have degree at least 2 for each $j>\ell$. Take $W=\left\langle r_{1}, \ldots, r_{\ell}\right\rangle_{K}$. Then we have

$$
\tilde{q}=\tilde{r}_{\ell+1} \tilde{s}_{\ell+1}+\cdots+\tilde{r}_{k} \tilde{s}_{k} .
$$

Now pair with any $x \in(V / W)^{*}$ and use the Leibniz rule on the right to obtain $S(\langle x, \tilde{q}\rangle) \leq 2(k-\ell)$.

Example 7. Let $d \in \mathbb{Z}_{\geq 2}$. Let $x_{1}, \ldots, x_{n}$ be a basis of $\mathbb{C}^{n}$ and write $q_{d, n}:=x_{1}^{d}+\cdots+x_{n}^{d}$. Then we claim that $S\left(q_{d, n}\right) \rightarrow \infty$ for $n \rightarrow \infty$. First, since $q_{d, n-1}$ is obtained from $q_{d, n}$ by setting $x_{n}$ equal to zero, $S\left(q_{d, n}\right)_{n}$ is a weakly increasing sequence, and it suffices to prove that it is unbounded. We prove this by induction on $d$. For $d=2$ we have $S\left(q_{2, n}\right)=\lceil n / 2\rceil$ by elementary linear algebra. Let $d \geq 3$ and assume the claim holds for $d-1$. Suppose $S\left(q_{d, n}\right) \leq k$, and let $\ell \leq k$ and $W \subseteq \mathbb{C}^{n}$ be as in Lemma 6 . Then

$$
\tilde{q}_{d, n}=\tilde{x}_{1}^{d}+\cdots+\tilde{x}_{n}^{d}
$$

where, without loss of generality, we may assume that $\tilde{x}_{1}, \ldots, \tilde{x}_{n-\ell}$ are a basis of $\mathbb{C}^{n} / W$. It follows that every directional derivative of

$$
\tilde{x}_{1}^{d}+\cdots+\tilde{x}_{n-\ell}^{d}=\tilde{q}_{d, n}-\left(\tilde{x}_{n-\ell+1}^{d}+\cdots+\tilde{x}_{n}^{d}\right)
$$

has strength at most $2(k-\ell)+\ell=2 k-\ell \leq 2 k$. The left-hand side is just $q_{d, n-\ell}$ in disguise. But $\left(\sum_{i} \frac{\partial}{\partial x_{i}}\right) q_{d, n-\ell}=d q_{d-1, n-\ell}$, so $S\left(q_{d-1, n-k}\right) \leq S\left(q_{d-1, n-\ell}\right) \leq 2 k$. Hence if the sequence $\left(S\left(q_{d, n}\right)\right)_{n}$ were bounded from above, so was the sequence $\left(S\left(q_{d-1, n}\right)\right)_{n}$, and 
this contradiction proves the claim. An immediate consequence is that the infinite series $x_{1}^{d}+x_{2}^{d}+\cdots$ has infinite strength.

Equip $S_{\infty}^{d}$ with the inverse limit topology. The group $\mathrm{GL}_{\infty}:=\bigcup_{n} \mathrm{GL}_{n}(K)$ acts on $S_{\infty}^{d}$ by homeomorphisms. The following is an immediate consequence of Theorem 4 .

Corollary 8. Fix $d \in \mathbb{Z}_{\geq 2}$ and assume that $K$ is an infinite and perfect field with char $K=0$ or char $K>d$. Then the $\mathrm{GL}_{\infty}$-orbit of any element $q \in S_{\infty}^{d}$ of infinite strength is dense in $S_{\infty}^{d}$.

1.5. Strength of tensors and alternating tensors. Let $V \in$ Vec. For alternating tensors $q \in \bigwedge^{d} V$, the strength $S(q)$ is defined as the minimal number $k$ of terms in any decomposition of the form

$$
q=r_{1} \wedge s_{1}+\cdots+r_{k} \wedge s_{k}
$$

where $r_{i} \in \bigwedge^{e_{i}} V$ and $s_{i} \in \bigwedge^{d-e_{i}} V$ for suitable natural numbers $e_{i} \in\{1, \ldots, d-1\}$. By taking all $e_{i}$ equal to 1 , and using standard properties of the wedge product, we obtain the bound $S(q) \leq \operatorname{dim} V-d+1$.

Next let $V=\left(V_{1}, \ldots, V_{d}\right) \in \operatorname{Vec}^{d}$ and define $T^{d} V:=V_{1} \otimes \cdots \otimes V_{d}$. For tensors $q \in T^{d} V$, the strength $S(q)$ is defined as the minimal number $k$ of terms in any decomposition of the form

$$
q=r_{1} \otimes s_{1}+\cdots+r_{k} \otimes s_{k}
$$

where $r_{i} \in \bigotimes_{j \in J_{i}} V_{j}$ and $s_{i} \in \bigotimes_{j \in[d] \backslash V_{i}} V_{j}$ for suitable non-empty subsets $J_{i} \subsetneq[d]$. By taking all $J_{i}$ equal to $\{\ell\}$, we obtain the bound $S(q) \leq \operatorname{dim} V_{\ell}$ for any $\ell \in[d]$.

1.6. Closed subsets of $\bigwedge^{d}$ and $T^{d}$. For all $V \in$ Vec, we equip $\wedge^{d} V$ with the Zariski topology. A closed subset of $\wedge^{d}$ is a rule $X$ that assigns to every $V \in$ Vec a closed subset $X(V)$ of $\bigwedge^{d} V$ in such a manner that for any $\varphi \in \operatorname{Hom}_{V e c}(V, W)$ the alternating power $\Lambda^{d} \varphi$ maps $X(V)$ into $X(W)$. In particular, the subset $X(V)$ is stable under the group $\mathrm{GL}(V)$ and if the map $\varphi$ is surjective, then $X(V)$ maps onto $X(W)$.

Similarly, for $V=\left(V_{1}, \ldots, V_{d}\right) \in \operatorname{Vec}^{d}$, we equip $T^{d} V$ with the Zariski topology. A closed subset of $T^{d}$ is a rule $X$ that assigns to every $V \in \operatorname{Vec}^{d}$ a closed subset $X(V)$ of $T^{d} V$ in such a manner that for any $d$-tuple of linear maps $\varphi_{i} \in \operatorname{Hom}_{V e c}\left(V_{i}, W_{i}\right)$ the tensor product $T^{d} \varphi:=\varphi_{1} \otimes \cdots \otimes \varphi_{d}$ maps $X(V)$ into $X(W)$. In particular, the subset $X(V)$ is stable under the group $\mathrm{GL}(V):=\mathrm{GL}\left(V_{1}\right) \times \cdots \times \mathrm{GL}\left(V_{d}\right)$ and if the maps $\varphi_{i}$ are all surjective, then $X(V)$ maps onto $X(W)$.

\subsection{Main theorem for tensors and alternating tensors.}

Theorem 9. Fix $d \in \mathbb{Z}_{\geq 2}$ and let $K$ be a perfect and infinite field with char $K=0$ or char $K>d$. Then for any closed $X \subsetneq \Lambda^{d}$ there exists an $N$ such that for all finitedimensional vector spaces $V$ the strength of all elements in $X(V)$ is at most $N$.

Theorem 10. Fix $d \in \mathbb{Z}_{\geq 2}$ and let $K$ be a perfect and infinite field. Then for any closed $X \subsetneq T^{d}$ there exists an $N$ such that for all finite-dimensional vector spaces $V_{1}, \ldots, V_{d}$ the strength of all elements in $X\left(V_{1}, \ldots, V_{d}\right)$ is at most $N$.

Both of these theorems have reformulations in terms of suitable projective limits as in Corollary 8 , we leave these to the reader. 
1.8. A version over $\mathbb{Z}$. Theorems 4,9 and 10 require that $K$ be fixed in advance, and allow for the closed subsets of $S^{d}, \wedge^{d}, T^{d}$ to be defined by equations specific to $K$. The price that we pay for this generality is that we need to require $K$ to be perfect and infinite, and that the values of $N$ in these theorems depend on $K$.

Indeed, in the proofs, perfectness of the field is used to ensure that a squarefree nonzero polynomial has some nonzero directional derivative; and infiniteness of the field is used to ensure that if some polynomial in $t$ vanishes for all $t \in K$, then the coefficients of all monomials $t^{d}$ vanish. We can get around both of these restrictions by working only with tensor properties defined over $\mathbb{Z}$ before specialising to $K$.

Let $\mathrm{Vec}_{\mathbb{Z}}$ be the category of finite-rank free $\mathbb{Z}$-modules with $\mathbb{Z}$-linear maps. Every object $V \in V c_{\mathbb{Z}}$ gives rise to an affine scheme, the spectrum of the symmetric algebra (over $\mathbb{Z}$ ) on the module dual to $V$. By abuse of notation, we write $V$ for this scheme, as well. The scheme of a product $V \times W$ is canonically isomorphic to the product of the schemes, and a $\varphi \in \operatorname{Hom}_{\mathrm{Vec}_{\mathbb{Z}}}(V, W)$ determines a morphism of schemes $V \rightarrow W$.

A module $V \in \operatorname{Vec}_{\mathbb{Z}}$ has a symmetric power $S_{\mathbb{Z}}^{d} V \in \operatorname{Vec}_{\mathbb{Z}}$ characterised by the usual universal property. A closed subscheme of $S_{\mathbb{Z}}^{d}$ is a rule $X_{\mathbb{Z}}$ that assigns to each $V \in \operatorname{Vec}_{\mathbb{Z}}$ a closed subscheme of $S_{\mathbb{Z}}^{d} V$ in such a manner that for $V, W \in \operatorname{Vec}_{\mathbb{Z}}$ and $\varphi \in \operatorname{Hom}_{V_{\text {ec }}}(V, W)$ the morphism $S_{\mathbb{Z}}^{d} \varphi$ maps $X_{\mathbb{Z}}(V)$ into $X_{\mathbb{Z}}(W)$. This is equivalent to the condition that the morphism of schemes determined by

$$
S_{\mathbb{Z}}^{d} V \times \operatorname{Hom}_{V_{\text {ec }}}(V, W) \rightarrow S_{\mathbb{Z}}^{d} W, \quad\left(v_{1} \cdots v_{d}, \varphi\right) \mapsto \varphi\left(v_{1}\right) \cdots \varphi\left(v_{d}\right)
$$

maps $X_{\mathbb{Z}}(V) \times \operatorname{Hom}_{V_{\text {ec }}}(V, W)$ into $X_{\mathbb{Z}}(W)$.

In terms of equations this means the following: Suppose that $V=\mathbb{Z}^{m}$ and $W=\mathbb{Z}^{n}$, let $f$ be any polynomial in the $\left(\begin{array}{c}n-1+d \\ d\end{array}\right)$ standard coordinates on $S_{\mathbb{Z}}^{d} W$ with coefficients in $\mathbb{Z}$, and let $\varphi$ be an $n \times m$-matrix whose entries $\varphi_{i j}$ are variables. Then one can expand $f \circ S_{\mathbb{Z}}^{d} \varphi$ as a polynomial $\sum_{\alpha \in \mathbb{Z}_{\geq 0}^{n \times m}} c_{\alpha} \varphi^{\alpha}$ in the $\varphi_{i j}$ whose coefficients $c_{\alpha}$ are polynomials in the $\left(\begin{array}{c}m-1+d \\ d\end{array}\right)$ standard coordinates on $S_{\mathbb{Z}}^{d} V$. The condition above says that if $f$ is in the ideal of $X_{\mathbb{Z}}(W)$, then all the $c_{\alpha}$ lie in the ideal of $X_{\mathbb{Z}}(V)$.

If $X_{\mathbb{Z}}$ is a closed subscheme of $S_{\mathbb{Z}}^{d}$, then for each field $K$ we obtain a closed subset $X_{K}$ of $S^{d}=S_{K}^{d}$ as follows: for $V \in V e c=V c_{K}$ choose any linear isomorphism $\varphi: V \rightarrow K^{n}$, and let $X_{K}(V)$ be the preimage under $S^{d} \varphi$ of the set of $K$-valued points of the scheme $X\left(\mathbb{Z}^{n}\right) \subseteq S^{d} \mathbb{Z}^{n}$.

Remark 11. For all field extensions $K \subseteq L$, we have

$$
X_{L}\left(V \otimes_{K} L\right) \cap S^{d} V=X_{K}(V)
$$

for all vector spaces $V \in \operatorname{Vec}_{K}$.

Theorem 12. Let $d \in \mathbb{Z}_{\geq 2}$ and let $X_{\mathbb{Z}}$ be a closed subscheme of $S_{\mathbb{Z}}^{d}$. Then there exists an $N \in \mathbb{Z}_{\geq 0}$ such that the following holds:

( $\dagger$ Let $K$ be any field with char $K=0$ or char $K>d$ such that $X_{K} \subsetneq S_{K}^{d}$. Then for all $V \in \operatorname{Vec}_{K}$ the strength of all elements in $X_{K}(V)$ is at most $N$.

Example 13 (Examples 2 and 3 revisited). Let $V \in \operatorname{Vec}_{\mathbb{Z}}$. For a sequence $e_{1}, \ldots, e_{k}$ of integers in $\{1, \ldots, d-1\}$ there is a morphism of schemes

$$
\prod_{i=1}^{k}\left(S_{\mathbb{Z}}^{e_{i}} V \times S_{\mathbb{Z}}^{d-e_{i}} V\right) \rightarrow S_{\mathbb{Z}}^{d} V, \quad\left(\left(r_{1}, s_{1}\right), \ldots,\left(r_{k}, s_{k}\right)\right) \mapsto r_{1} s_{1}+\cdots+r_{k} s_{k} .
$$


We define $Y_{d, k, \mathbb{Z}}(V)$ as its scheme-theoretic image, i.e., as the closed subscheme defined by the kernel of the homomorphism of rings in the opposite direction. A straightforward verification shows that $Y_{d, k, \mathbb{Z}}$ is a closed subscheme of $S_{\mathbb{Z}}^{d}$. A similar construction yields a $\mathbb{Z}$-version $Z_{d, k, \mathbb{Z}}$ of $Z_{d, k}$. The previous theorem applied to these closed subschemes yields a bound $N$ on the strength of elements of $Z_{d, k}(W), W \in$ $\operatorname{Vec}_{K}$ that is independent of $W$ and $K$ ( $K$ may now be finite or non-perfect, but must satisfy the conditions on the characteristic). This yields the main result of [KZ18].

The $\mathbb{Z}$-constructions in this subsection have analogues for the polynomial functors $\Lambda^{d}$ and $T^{d}$, and the analogues of Theorems 9 and 10 also hold over $\mathbb{Z}$.

Theorem 14. Let $X$ be a closed subscheme of $\bigwedge_{\mathbb{Z}}^{d}$. Then there exists an $N \in \mathbb{Z}_{\geq 0}$ such that the following holds:

(†) Let $K$ be any field with $\operatorname{char} K=0$ or char $K>d$ such that $X_{K} \subsetneq \bigwedge_{K}^{d}$. Then for all $V \in \operatorname{Vec}_{K}$ and all $q \in X_{K}(V)$ we have $S(q) \leq N$.

Theorem 15. Let $X$ be a closed subscheme of $T_{\mathbb{Z}}^{d}$. Then there exists an $N \in \mathbb{Z}_{\geq 0}$ such that the following holds:

(†) Let $K$ be any field such that $X_{K} \subsetneq T_{K}^{d}$. Then for all $V \in \operatorname{Vec}_{K}^{d}$ and all $q \in X_{K}(V)$ we have $S(q) \leq N$.

1.9. Relation to previous work. For $d=3$, our Corollary 8 is proved in [DES17, Theorem 1.7]. For $Z_{d, k, \mathbb{Z}}$, our Theorem 4 (and indeed, reading carefully, Theorem 12 ) is proved in [KZ18] using Gowers norms-interestingly, this requires a detour via finite fields to prove the theorem for $K=\mathbb{C}$. Our proof is quite different, and follows the second author's technique from [Dra17].

Unlike for Waring rank and tensor rank, there seems to be very little literature on equations for the varieties $Y_{d, k}(V)$ of polynomials of bounded strength. For $d=3$ and $k=1$ the ideal is generated by 35 polynomials of degree 8 [Chi02].

1.10. Organisation. In Section 2 we prove Theorem 4 In Sections 3 and 4 we adapt this proof to the case of alternating and ordinary tensors, respectively. In Remarks 23, 29, and 35 we give explicit (though probably non-optimal!) values for $N$ from Theorems 4, 9, and 10, These are used in Section 5, where we prove Theorem 12 .

Acknowledgments. The authors thank Professor Kazhdan for the heads-up on [KZ18] and stimulating e-mail discussions. The second author thanks the Institut Mittag-Leffler for their hospitality during this project.

\section{PROOF FOR POLYNOMIALS}

In this section we prove Theorem 4 By assumption, there exists a $U \in$ Vec such that $X(U) \subsetneq S^{d} U$. We fix this $U$ throughout the proof. The bound $N$ that we will obtain depends only on $d$ and $\operatorname{dim} U$, see Remark 23

2.1. Irreducibility. The following lemma is a standard fact from representation theory. Recall that char $K=0$ or char $K>d$. This implies that for any $V \in \operatorname{Vec}$ the $\mathrm{GL}(V)$-module $S^{d}\left(V^{*}\right)$ is isomorphic to $\left(S^{d} V\right)^{*}$. 
Lemma 16. For each $V \in \mathrm{Vec}$, the $\mathrm{GL}(V)$-module $S^{d} V$ is irreducible and linearly spanned by its closed subvariety $P:=\left\{v^{d} \mid v \in V\right\} \subseteq S^{d} V$. Furthermore, any $\mathrm{GL}(V)$-equivariant polynomial map from $V$ into $a \mathrm{GL}(V)$-module $N$ on which $t 1_{V}$ acts via multiplication with $t^{d}$ factors as $V \rightarrow S^{d} V, v \mapsto v^{d}$ and a unique $\mathrm{GL}(V)$-equivariant linear map $S^{d} V \rightarrow N$.

2.2. Homogeneity. We equip the coordinate ring $K\left[S^{d} V\right]$ with the $d \cdot \mathbb{Z}_{\geq 0}$-grading in which the elements of $\left(S^{d} V\right)^{*}$ have degree $d$. For any closed $X \subseteq S^{d}$ we find, from the fact that $X(V)$ is $\mathrm{GL}(V)$-stable, that the ideal $\mathcal{I}(X(V)) \subseteq K\left[S^{d} V\right]$ is $\mathrm{GL}(V)$-stable and in particular homogeneous. We define

$$
\delta_{X}:=\min _{f \in \mathcal{I}(X(U)) \backslash\{0\}} \operatorname{deg} f \in d \mathbb{Z}_{\geq 0} .
$$

2.3. Induction. If $\delta_{X}=0$, then $\mathcal{I}(X(U))$ contains a nonzero constant polynomial, so that $X(U)=\emptyset$. For any $V \in$ Vec, the $d$-th symmetric power of the zero map $0_{V \rightarrow U}: V \rightarrow U$ maps $X(V)$ into $X(U)$, hence all $X(V)$ are empty. Hence in Theorem 4 we may take $N=0$. We proceed by induction, assuming that $\delta_{X}>0$ and that the theorem holds for all $Y \subseteq S^{d}$ with $Y(U) \subsetneq S^{d} U$ and $\delta_{Y}<\delta_{X}$.

2.4. Derivative. Let $f \in \mathcal{I}(X(U)) \backslash\{0\}$ be homogeneous of degree $\delta_{X}$. By minimality of $\delta_{X}$ and perfectness of $K$, there exists an $r \in S^{d} U$ such that the directional derivative $h:=\frac{\partial f}{\partial r}$ is not the zero polynomial. By Lemma 16, $S^{d} U$ is spanned by $d$-th powers, so we may further assume that $r=u^{d}$ for some $u \in U$.

We define the closed subset $Y \subsetneq S^{d}$ by

$$
Y(V):=\left\{q \in X(V) \mid \forall \varphi \in \operatorname{Hom}_{\mathrm{Vec}}(V, U): h\left(\left(S^{d} \varphi\right) q\right)=0\right\} .
$$

Now $\delta_{Y} \leq \operatorname{deg} h=\operatorname{deg} f-d<\operatorname{deg} f$, so by the induction hypothesis the theorem holds for $Y$. We define

$$
Z(V):=X(V) \backslash Y(V),
$$

and set out to prove that all elements in $Z(V)$ have bounded strength independent of $V$.

2.5. Shifting. For $V \in$ Vec we define

$$
\begin{aligned}
& P^{\prime}(V):=S^{d}(U \oplus V)=\bigoplus_{i=0}^{d} S^{d-i} U \otimes S^{i} V, \\
& X^{\prime}(V):=X(U \oplus V) \subseteq P^{\prime}(V), \\
& Z^{\prime}(V):=\left\{q \in X^{\prime}(V) \mid h\left(\left(S^{d}\left(1_{U} \oplus 0_{V \rightarrow U}\right)\right) q\right) \neq 0\right\} ;
\end{aligned}
$$

the notation is chosen compatible with [Dra17]. We think of $P^{\prime}(V), X^{\prime}(V)$ as varieties over $S^{d} U, X(U)$, respectively, via the linear map $S^{d}\left(1_{U} \oplus 0_{V \rightarrow U}\right)$. Accordingly, by slight abuse of notation, we will write $h$ for $h \circ S^{d}\left(1_{U} \oplus 0_{V \rightarrow U}\right)$.

Lemma 17. We have

$$
Z(U \oplus V)=\bigcup_{g \in \mathrm{GL}(U \oplus V)} g Z^{\prime}(V) .
$$

In particular, $\sup _{q \in Z(U \oplus V)} S(q)=\sup _{q \in Z^{\prime}(V)} S(q)$. 
Proof. First, we have $Z(U \oplus V) \supseteq Z^{\prime}(V)$, and since the left-hand side is GL $(U \oplus V)$ stable, the inclusion $\supseteq$ follows. Conversely, if $q \in Z(U \oplus V)$, then there exists a linear $\operatorname{map} \varphi: U \oplus V \rightarrow U$ for which $h\left(\left(S^{d} \varphi\right) q\right) \neq 0$. Since this is an open condition on $\varphi$, we may further assume that $\varphi$ has full rank. Then for a suitable $g \in \mathrm{GL}(U \oplus V)$ we find that $\varphi=\left(1_{U} \oplus 0_{V \rightarrow U}\right) \circ g$. Accordingly,

$$
h(g q)=h\left(\left(S^{d}\left(1_{U} \oplus 0_{V \rightarrow U}\right)\right)\left(S^{d} g\right) q\right)=h\left(\left(S^{d} \varphi\right) q\right) \neq 0
$$

and hence $g q \in Z^{\prime}(V)$.

Lemma 18. We have

$$
\sup _{V \in \operatorname{Vec}} \sup _{f \in X(V)} S(f)=\sup _{V \in \operatorname{Vec}} \sup \left\{\sup _{f \in Y(V)} S(f), \sup _{f \in Z^{\prime}(V)} S(f)\right\} .
$$

Proof. The same statement with $Z^{\prime}(V)$ replaced by $Z(V)$ is obvious given the fact that $X(V)=Y(V) \cup Z(V)$. The map $S^{d}\left(0_{V \rightarrow U}, 1_{V}\right)$ maps $Z(V)$ into $Z(U \oplus V)$ and is easily seen to preserve the strength. By the previous lemma

$$
\sup _{f \in Z^{\prime}(V)} S(f)=\sup _{f \in Z(U \oplus V)} S(f)
$$

and so the statement follows.

So it suffices to show that elements in $Z^{\prime}(V)$ have bounded strength.

\subsection{Chopping.}

Lemma 19. For $q \in P^{\prime}(V)$ write $q=q_{0}+\cdots+q_{d}$ with $q_{i} \in S^{d-i} U \otimes S^{i} V$. Then

$$
S(q) \leq \operatorname{dim} U+S\left(q_{d}\right) .
$$

Proof. Note that $q_{0}+\ldots+q_{d-1}$ is in the image of the map

$$
\begin{aligned}
U \otimes S^{d-1}(U \oplus V) & \rightarrow S^{d}(U \oplus V) \\
r \otimes S & \mapsto r s
\end{aligned}
$$

and hence has strength at most $\operatorname{dim} U$. Now, strength is subadditive, so

$$
S(q) \leq S\left(q_{0}+\ldots+q_{d-1}\right)+S\left(q_{d}\right) \leq \operatorname{dim} U+S\left(q_{d}\right) .
$$

So, as $U$ is fixed, it suffices to prove that for $V$ ranging through Vec and $q$ ranging through $Z^{\prime}(V)$ the component $q_{d}$ has bounded strength.

2.7. Embedding. Define

$$
Q^{\prime}(V):=P^{\prime}(V) / S^{d} V=\bigoplus_{i=0}^{d-1} S^{d-i} U \otimes S^{i} V
$$

and write $\pi(V): P^{\prime}(V) \rightarrow Q^{\prime}(V)$ for the natural projection. Let

$$
B(V):=\left\{q \in Q^{\prime}(V) \mid h(q) \neq 0\right\}=\left\{\left(q_{0}, \ldots, q_{d-1}\right) \in Q^{\prime}(V) \mid h\left(q_{0}\right) \neq 0\right\},
$$

an open set in $Q^{\prime}(V)$. Then $\pi(V)$ maps $Z^{\prime}(V)$ into $B(V)$, and by [Dra17, Lemma 7] and Lemma 16, we have the following.

Lemma 20. The map $\pi(V)$ restricts to a closed embedding $Z^{\prime}(V) \rightarrow B(V)$.

We will not actually use this lemma, but we will use its proof method. 
2.8. An equivariant map back. We construct a suitable map opposite to the embedding of Lemma 20 .

Lemma 21. There exists a GL(V)-equivariant polynomial map $\Psi: Q^{\prime}(V) \rightarrow S^{d} V$ such that, for all $q=\left(q_{0}, \ldots, q_{d}\right) \in Z^{\prime}(V), q_{d}$ is a scalar multiple of $\Psi\left(q_{0}, \ldots, q_{d-1}\right)$.

Proof. For $x \in V^{*}$, let $\varphi_{x}: V \rightarrow U$ be the linear map $v \mapsto x(v) u$; here $u$ is the vector used in the definition of $h$. Note that $x \mapsto \varphi_{x}$ is a GL(V)-equivariant linear map $V^{*} \rightarrow \operatorname{Hom}_{\mathrm{Vec}}(V, U)$.

For every $t \in K$ define the linear map $\Phi_{x}(t):=S^{d}\left(1_{U} \oplus t \varphi_{x}\right): P^{\prime}(V) \rightarrow S^{d} U$. The restriction of $\Phi_{x}(t)$ to the summand $S^{d-i} U \otimes S^{i} V$ equals $t^{i} \Phi_{x, i}$ where $\Phi_{x, i}$ is the composition of $S^{d-i} 1_{U} \otimes S^{i} \varphi_{x}: S^{d-i} U \otimes S^{i} V \rightarrow S^{d-i} U \otimes S^{i} U$ and the multiplication map into $S^{d} U$. In particular, $\Phi_{x, 0}$ is the identity on $S^{d} U$ and $\Phi_{x, d}$ is the linear map $S^{d} V \rightarrow S^{d} U, q_{d} \mapsto x^{d}\left(q_{d}\right) u^{d}$. Note that $V^{*} \rightarrow \operatorname{Hom}_{\mathrm{Vec}}\left(S^{d-i} U \otimes S^{i} V, S^{d} U\right), x \mapsto \Phi_{x, i}$ is a $\mathrm{GL}(V)$-equivariant polynomial map of (ordinary) degree $i$.

The functoriality of $X$ implies that $\Phi_{x}(t) X^{\prime}(V) \subseteq X(U)$. In particular, the pullback of $f$ along $\Phi_{x}(t)$ to $P^{\prime}(V)$ vanishes on $X^{\prime}(V)$. Take $q=\left(q_{0}, \ldots, q_{d}\right) \in P^{\prime}(V)$. Then

$$
f\left(\Phi_{x}(t)\left(q_{0}+q_{1}+\cdots+q_{d-1}+q_{d}\right)\right)=f\left(q_{0}+t \Phi_{x, 1} q_{1}+\cdots t^{d-1} \Phi_{x, d-1} q_{d-1}+t^{d} x^{d}\left(q_{d}\right) u^{d}\right),
$$

and this vanishes for $q \in X^{\prime}(V)$. In particular, the coefficient of $t^{d}$ in the Taylor expansion of this expression vanishes for $q \in X^{\prime}(V)$. This coefficient equals

$$
x^{d}\left(q_{d}\right) \frac{\partial f}{\partial u^{d}}\left(q_{0}\right)+\Psi\left(x, q_{0}, \ldots, q_{d-1}\right)=x^{d}\left(q_{d}\right) h\left(q_{0}\right)+\Psi\left(x, q_{0}, \ldots, q_{d-1}\right) .
$$

where the function $\Psi: V^{*} \times Q^{\prime}(V) \mapsto K$ is GL(V)-invariant, and homogeneous of degree $d$ in its first argument $x$.

For $q \in Z^{\prime}(V)$ we have $h\left(q_{0}\right) \neq 0$ and hence

$$
x^{d}\left(q_{d}\right)=-\frac{1}{h\left(q_{0}\right)} \Psi\left(x, q_{0}, \ldots, q_{d-1}\right) .
$$

By Lemma 16 the space $\left(S^{d} V\right)^{*}$ of coordinates on $S^{d}$ is spanned by the $x^{d}, x \in V^{*}$, and this shows that $Z^{\prime}(V) \rightarrow B(V)$ is a closed embedding. But it yields more: by Lemma 16, $\Psi$ factors as

$$
\begin{aligned}
V^{*} \times Q^{\prime}(V) & \rightarrow S^{d} V^{*} \times Q^{\prime}(V) \\
\left(x, q^{\prime}\right) & \mapsto\left(x^{d}, q^{\prime}\right)
\end{aligned}
$$

and a unique GL(V)-invariant map $S^{d} V^{*} \times Q^{\prime}(V) \rightarrow K$. We denote the latter map also by $\Psi$, which is now linear in its first argument. If we re-interpret $\Psi$ as $\mathrm{GL}(V)$-equivariant polynomial map $Q^{\prime}(V) \rightarrow S^{d} V$, then for $q \in Z^{\prime}(V)$ we have

$$
q_{d}=-\frac{1}{h\left(q_{0}\right)} \Psi\left(q_{0}, \ldots, q_{d-1}\right) .
$$

In particular, for $q \in Z^{\prime}(V)$ we have $q_{d} \in \operatorname{im} \Psi$.

2.9. Covariants. A covariant of $Q^{\prime}(V)$ (of order $S^{d} V$, the only order that we will use) is a $\mathrm{GL}(V)$-equivariant polynomial map $Q^{\prime}(V) \rightarrow S^{d} V$. So the map $\Psi$ constructed 
in 2.8 is a covariant. For each integer $i \in[d-1]$, choose a basis $u_{i, 1}, \ldots, u_{i, n_{i}}$ of $S^{d-i} U$. Then the map

$$
\begin{aligned}
\Phi: \bigoplus_{i=0}^{d-i}\left(S^{i} V\right)^{\oplus n_{i}} & \rightarrow \bigoplus_{i=1}^{d-1} S^{d-i} U \otimes S^{i} V \\
\left(w_{i, j}\right)_{i, j} & \mapsto\left(\sum_{j=1}^{n_{i}} u_{i, j} \otimes w_{i, j}\right)_{i=1}^{d-1}
\end{aligned}
$$

is a $\mathrm{GL}(V)$-equivariant isomorphism and the following lemma holds.

Lemma 22. Let $\Psi: Q^{\prime}(V) \rightarrow S^{d} V$ be a covariant. Then the composition $\Psi \circ\left(\operatorname{id}_{S^{d} U}, \Phi\right)$ is of the form

$$
\begin{aligned}
S^{d} U \oplus \bigoplus_{i=1}^{d-1}\left(S^{i} V\right)^{\oplus n_{i}} & \rightarrow S^{d} V \\
\left(q,\left(w_{i, j}\right)_{i, j}\right) & \mapsto \sum_{\substack{\alpha_{i, j} \in \mathbb{Z}_{\geq 0} \\
\sum_{i=1}^{d-1} \sum_{j=1}^{n_{i}} i \cdot \alpha_{i, j}=d}} p_{\alpha}(q) \cdot \prod_{i=1}^{d-1} \prod_{j=1}^{n_{i}} w_{i, j}^{\alpha_{i, j}}
\end{aligned}
$$

for some polynomial functions $p_{\alpha}: S^{d} U \rightarrow K$.

Proof. Polynomial GL(V)-equivariant maps

$$
S^{d} U \oplus \bigoplus_{i=1}^{d-1}\left(S^{i} V\right)^{\oplus n_{i}} \rightarrow S^{d} V
$$

correspond one-to-one to linear $\mathrm{GL}(V)$-equivariant maps

$$
K\left[S^{d} U\right] \otimes \bigoplus_{\substack{\alpha_{i,} \in \mathbb{Z}_{\geq 0} \\ \sum_{i=1}^{d-1} \sum_{j=1}^{n_{i}} \cdot \alpha_{i, j}=d}} \bigotimes_{i=1}^{d-1} \bigotimes_{j=1}^{n_{i}} S^{\alpha_{i, j}} S^{i} V \rightarrow S^{d} V
$$

by the universal properties of tensor products and symmetric powers. For each $\alpha$, the vector space

$$
\operatorname{Hom}_{\mathrm{GL}(V)}\left(\bigotimes_{i=1}^{d-1} \bigotimes_{j=1}^{n_{i}} S^{\alpha_{i, j}} S^{i} V, S^{d} V\right)
$$

is one-dimensional and consists of multiples of the homomorphism $\ell_{\alpha}$ sending

$$
\bigotimes_{i=1}^{d-1} \bigotimes_{j=1}^{n_{i}} w_{i, j, 1} \cdots \cdots w_{i, j, \alpha_{i, j}} \mapsto \prod_{i=1}^{d-1} \prod_{j=1}^{n_{i}} w_{i, j, 1} \cdots \cdots w_{i, j, \alpha_{i, j}}
$$

Hence the set of linear GL(V)-equivariant maps

$$
K\left[S^{d} U\right] \otimes \bigoplus_{\substack{\alpha_{i, j} \in \mathbb{Z}_{\geq 0} \\ \sum_{i=1}^{d-1} \sum_{j=1}^{n_{i}} \cdot \cdot \alpha_{i, j}=d}} \bigotimes_{i=1}^{d-1} \bigotimes_{j=1}^{n_{i}} S^{\alpha_{i, j}} S^{i} V \rightarrow S^{d} V
$$

is spanned as a $K\left[S^{d} U\right]$-module by the set of all $\ell_{\alpha}$. The corresponding statement for polynomial GL(V)-equivariant maps is the statement of the lemma. 


\subsection{Conclusion of the proof.}

Proof of Theorem 4 By the induction hypothesis and Lemma 18, to bound the strength of elements of $X(V)$ for all $V \in$ Vec it suffices to bound the strength of elements of $Z^{\prime}(V)$ for all $V \in$ Vec. By Lemma19, it suffices to bound the strength of $q_{d}$ over all $q=\left(q_{0}, \ldots, q_{d}\right) \in Z^{\prime}(V)$. By Lemma 21, we know that such a $q_{d}$ is contained in the image of a covariant. So using Lemma22, we see that $q_{d}$ is a linear combination of products of polynomials $w_{i, j} \in S^{i} V$ where $i$ ranges over [ $\left.d-1\right]$ and $j$ ranges over [dim $S^{d-i} U$ ]. Since each of those products has degree $d$ and since, for each pair $(i, j)$, the polynomial $w_{i, j}$ has degree $i$, we see that each of the products is divisible by $w_{i, j}$ for some $i \leq d / 2$. We find that the strength of $q_{d}$ is at most

$$
\#\left\{w_{i, j} \mid i \in\{1, \ldots,\lfloor d / 2\rfloor\}, j \in\left[\operatorname{dim} S^{d-i} U\right]\right\} \leq \sum_{i=1}^{\lfloor d / 2\rfloor} \operatorname{dim} S^{d-i} U
$$

and this bounds the strength of $q_{d}$ independently of $V$.

Remark 23. It follows from the induction that $N$ from Theorem 4 can be taken equal to

$$
\operatorname{dim} U+\sum_{i=1}^{\lfloor d / 2\rfloor} \operatorname{dim} S^{d-i} U
$$

\section{Proof For Alternating tensors}

In this section we adapt the proof from Section 2 to a proof of Theorem 9 for alternating tensors. Throughout this section, we assume that $\operatorname{char} K=0$ or char $K>d$, we will let $X$ be a closed subset of $\Lambda^{d}$ and $U$ a finite-dimensional vector space such that $X(U) \subsetneq \bigwedge^{d} U$. Note that $\operatorname{dim} U \geq d$ as $\bigwedge^{d} U \neq \emptyset$.

3.1. Irreducibility. Note that for any $V \in \operatorname{Vec}$ the $\operatorname{GL}(V)$-modules $\wedge^{d}\left(V^{*}\right)$ and $\left(\bigwedge^{d} V\right)^{*}$ are isomorphic. The analogue of Lemma 16 is as follows.

Lemma 24. For each $V \in \mathrm{Vec}$, the $\mathrm{GL}(V)$-module $\wedge^{d} V$ is irreducible and linearly spanned by its closed subvariety $P:=\left\{v_{1} \wedge \ldots \wedge v_{d} \mid v_{1}, \ldots, v_{d} \in V\right.$ linearly independent $\} \subseteq \wedge^{d} V$. Furthermore, any GL(V)-equivariant multilinear and alternating map from $V^{d}$ into a $\mathrm{GL}(V)$-module $N$ on which $t 1_{V}$ acts via multiplication with $t^{d}$ extends uniquely to a $\mathrm{GL}(V)$-equivariant linear map $\wedge^{d} V \rightarrow N$.

3.2. Homogeneity. We equip the coordinate ring $K\left[\wedge^{d} V\right]$ with the $d \cdot \mathbb{Z}_{\geq 0}$-grading in which the elements of $\left(\bigwedge^{d} V\right)^{*}$ have degree $d$. For any closed $X \subseteq \bigwedge^{d}$ we find, from the fact that $X(V)$ is stable under $\operatorname{GL}(V)$, that the ideal $\mathcal{I}(X(V)) \subseteq K\left[\wedge^{d} V\right]$ is $\mathrm{GL}(V)$-stable and in particular homogeneous. We define

$$
\delta_{X}:=\min _{f \in \mathcal{I}(X(U)) \backslash\{0\}} \operatorname{deg} f \in d \mathbb{Z}_{\geq 0} .
$$

3.3. Induction. If $\delta_{X}=0$, then we find that $X(V)=\emptyset$ for all $V \in$ Vec. We may therefore assume that $\delta_{X}>0$ and we proceed by induction, assuming that the theorem holds for all $Y \subseteq \bigwedge^{d}$ with $Y(U) \subsetneq \bigwedge^{d} U$ and $\delta_{Y}<\delta_{X}$. 
3.4. Derivative. Let $f \in \mathcal{I}(X(U)) \backslash\{0\}$ be a homogeneous polynomial of degree $\delta_{X}$. Then, there exists an $r \in \bigwedge^{d} U$ such that the directional derivative $h:=\frac{\partial f}{\partial r}$ is not the zero polynomial. By Lemma24 we may assume that $r=u_{1} \wedge \ldots \wedge u_{d}$ for some linearly independent $u_{1}, \ldots, u_{d} \in U$.

We define $Y \subsetneq \wedge^{d}$ by

$$
Y(V):=\left\{q \in X(V) \mid \forall \varphi \in \operatorname{Hom}_{V e c}(V, U): h\left(\left(\bigwedge^{d} \varphi\right) q\right)=0\right\}
$$

and note that, by the induction hypothesis, the theorem holds for $Y$. We define

$$
Z(V):=X(V) \backslash Y(V)
$$

and prove that all elements of $Z(V)$ have bounded strength independent of $V$.

3.5. Shifting. For $V \in$ Vec we define

$$
\begin{aligned}
& P^{\prime}(V):=\bigwedge^{d}(U \oplus V)=\bigoplus_{i=0}^{d} \bigwedge^{d-i} U \otimes \bigwedge^{i} V, \\
& X^{\prime}(V):=X(U \oplus V) \subseteq P^{\prime}(V), \\
& Z^{\prime}(V):=\left\{q \in X^{\prime}(V) \mid h\left(\left(\bigwedge^{d}\left(1_{U} \oplus 0_{V \rightarrow U}\right)\right) q\right) \neq 0\right\} .
\end{aligned}
$$

We think of $P^{\prime}(V), X^{\prime}(V)$ as varieties over $\wedge^{d} U, X(U)$, respectively, via the linear map $\bigwedge^{d}\left(1_{U} \oplus 0_{V \rightarrow U}\right)$ and we will write $h$ for $h \circ \bigwedge^{d}\left(1_{U} \oplus 0_{V \rightarrow U}\right)$.

Lemma 25. We have

$$
\sup _{V \in \operatorname{Vec}} \sup _{q \in X(V)} S(q)=\sup _{V \in \operatorname{Vec}} \sup \left\{\sup _{q \in Y(V)} S(q), \sup _{q \in Z^{\prime}(V)} S(q)\right\} .
$$

\subsection{Chopping.}

Lemma 26. For $q \in P^{\prime}(V)$ write $q=q_{0}+\ldots+q_{d}$ with $q_{i} \in \bigwedge^{d-i} U \otimes \bigwedge^{i} V$. Then

$$
S(q) \leq \operatorname{dim} U+S\left(q_{d}\right) .
$$

\subsection{Embedding. Define}

$$
Q^{\prime}(V):=P^{\prime}(V) / \bigwedge^{d} V=\bigoplus_{i=0}^{d-1} \bigwedge^{d-i} U \otimes \bigwedge^{i} V
$$

and write $\pi(V): P^{\prime}(V) \rightarrow Q^{\prime}(V)$ for the natural projection. Let

$$
B(V):=\left\{q \in Q^{\prime}(V) \mid h(t) \neq 0\right\}=\left\{\left(q_{0}, \ldots, q_{d-1}\right) \in Q^{\prime}(V) \mid h\left(q_{0}\right) \neq 0\right\},
$$

an open set in $Q^{\prime}(V)$. Then $\pi(V)$ maps $Z^{\prime}(V)$ into $B(V)$ (and this is a closed embedding by [Dra17, Lemma 7] and Lemma 24). 


\subsection{An equivariant map back.}

Lemma 27. There exists a GL(V)-equivariant polynomial map

$$
\Psi: Q^{\prime}(V) \rightarrow \wedge^{d} V
$$

such that, for all $q=\left(q_{0}, \ldots, q_{d}\right) \in Z^{\prime}(V), q_{d}$ is a scalar multiple of $\Psi\left(q_{0}, \ldots, q_{d-1}\right)$.

Proof. For $x=\left(x_{1}, \ldots, x_{d}\right) \in\left(V^{*}\right)^{d}$, let $\varphi_{x, j}: V \rightarrow U$ be the linear map $v \mapsto x_{j}(v) u_{j}$; here $u_{1}, \ldots, u_{d}$ are the vectors used in the definition of $h$. Note that $x \mapsto \varphi_{x, j}$ is a $\mathrm{GL}(V)$-equivariant linear map $\left(V^{*}\right)^{d} \rightarrow \operatorname{Hom}_{\mathrm{Vec}}(V, U)$. For all $t_{1}, \ldots, t_{d} \in K$ define the linear map

$$
\Phi_{x}(t):=\bigwedge^{d}\left(1_{U} \oplus \sum_{j=1}^{d} t_{j} \varphi_{x, j}\right): P^{\prime}(V) \rightarrow \bigwedge^{d} U .
$$

Denote the restriction of $\Phi_{x}(t)$ to the summand $\bigwedge^{d-i} U \otimes \bigwedge^{i} V$ by $\Phi_{x, i}$. Note that $\Phi_{x, 0}$ is the identity on $\wedge^{d} U$ and $\Phi_{x, d}$ is the linear map

$$
\begin{aligned}
\wedge^{d} V & \rightarrow \wedge^{d} U \\
v_{1} \wedge \ldots \wedge v_{d} & \mapsto t_{1} \ldots t_{d} \cdot\left(\sum_{j=1}^{d} \varphi_{x, j}\left(v_{1}\right)\right) \wedge \ldots \wedge\left(\sum_{j=1}^{d} \varphi_{x, j}\left(v_{d}\right)\right)
\end{aligned}
$$

where the latter is a multiple of $u_{1} \wedge \ldots \wedge u_{d}$. Also note that $x \mapsto \Phi_{x, i}$ is a $\operatorname{GL}(V)$ equivariant polynomial map of (ordinary) degree $i$ and that $x \mapsto \Phi_{x, d}$ is multilinear and alternating.

By functoriality of $X$, we have $\Phi_{x}(t)\left(X^{\prime}(V)\right) \subseteq X(U)$, and for $q=\left(q_{0}, \ldots, q_{d}\right) \in$ $P^{\prime}(V)$ we find that

$$
f\left(\Phi_{x}(t)\left(q_{0}+\ldots+q_{d}\right)\right)=f\left(q_{0}+\Phi_{x, 1} q_{1}+\ldots+\Phi_{x, d-1} q_{d-1}+t_{1} \ldots t_{d} \cdot\left(\bigwedge^{d} \sum_{j=1}^{d} \varphi_{x, j}\right) q_{d}\right),
$$

and this expression vanishes for $q \in X^{\prime}(V)$. The coefficient of $t_{1} \ldots t_{d}$ in the Taylor expansion of this expression equals

$$
h\left(q_{0}\right)\left(x_{1} \wedge \cdots \wedge x_{d}\right) q_{d}+\Psi\left(x, q_{0}, \ldots, q_{d-1}\right)
$$

where the function $\Psi:\left(V^{*}\right)^{d} \times Q^{\prime}(V) \rightarrow K$ is $\mathrm{GL}(V)$-invariant and multilinear in $\left(V^{*}\right)^{d}$. We note that for $q \in Z^{\prime}(V)$, we have $h\left(q_{0}\right) \neq 0$ by definition of $Z^{\prime}(V)$, and therefore

$$
\left(x_{1} \wedge \cdots \wedge x_{d}\right) q_{d}=-\frac{1}{h\left(q_{0}\right)} \Psi\left(x, q_{0}, \ldots, q_{d-1}\right) .
$$

The map $\Psi$ factors as

$$
\begin{aligned}
\left(V^{*}\right)^{d} \times Q^{\prime}(V) & \rightarrow\left(V^{*}\right)^{\otimes d} \times Q^{\prime}(V) \\
\left(x, q^{\prime}\right) & \mapsto\left(x_{1} \otimes \cdots \otimes x_{d}, q^{\prime}\right)
\end{aligned}
$$

and a unique $\mathrm{GL}(V)$-equivariant map $\left(V^{*}\right)^{\otimes d} \times Q^{\prime}(V) \rightarrow K$. If we re-interpret $\Psi$ as a $\mathrm{GL}(V)$-equivariant polynomial map $Q^{\prime}(V) \rightarrow V^{\otimes d}$ and compose the map with the projection $V^{\otimes d} \rightarrow \bigwedge^{d} V$, then we get a map $Q^{\prime}(V) \rightarrow \bigwedge^{d} V$ which we also denote by $\Psi$. We see that

$$
d ! \cdot q_{d}=-\frac{1}{h\left(q_{0}\right)} \Psi\left(q_{0}, \ldots, q_{d-1}\right) .
$$

for all $q \in Z^{\prime}(V)$, since

$$
\left(x_{1} \wedge \cdots \wedge x_{d}\right) q_{d}=\left(x_{1} \otimes \cdots \otimes x_{d}\right) \iota\left(q_{d}\right)
$$


where $\iota$ is the $\mathrm{GL}(V)$-equivariant map

$$
\begin{aligned}
\iota: \wedge^{d} V & \rightarrow V^{\otimes d} \\
v_{1} \wedge \cdots \wedge v_{d} & \mapsto \sum_{\sigma \in S_{d}} \operatorname{sgn}(\sigma) \cdot v_{\sigma(1)} \otimes \cdots \otimes v_{\sigma(d)}
\end{aligned}
$$

3.9. Covariants. A covariant of $Q^{\prime}(V)$ (of order $S^{d} V$, the only order that we will use) is a $\mathrm{GL}(V)$-equivariant polynomial map $Q^{\prime}(V) \rightarrow \bigwedge^{d} V$. So the map $\Psi$ constructed in 3.8 is a covariant. For each integer $i \in[d-1]$, choose a basis $u_{i, 1}, \ldots, u_{i, n_{i}}$ of $\wedge^{d-i} U$. Then the map

$$
\begin{aligned}
\Phi: \bigoplus_{i=0}^{d-i}\left(\bigwedge^{i} V\right)^{\oplus n_{i}} & \rightarrow \bigoplus_{i=1}^{d-1} \bigwedge^{d-i} U \otimes \bigwedge^{i} V \\
\left(w_{i, j}\right)_{i, j} & \mapsto\left(\sum_{j=1}^{n_{i}} u_{i, j} \otimes w_{i, j}\right)_{i=1}^{d-1}
\end{aligned}
$$

is a $\mathrm{GL}(V)$-equivariant isomorphism and the following lemma holds.

Lemma 28. Let $\Psi: Q^{\prime}(V) \rightarrow \wedge^{d} V$ be a covariant. Then the composition $\Psi \circ\left(\mathrm{id}_{\wedge^{d} U^{\prime}} \Phi\right)$ is of the form

$$
\begin{aligned}
\bigwedge^{d} U \oplus \bigoplus_{i=1}^{d-1}\left(\bigwedge^{i} V\right)^{\oplus n_{i}} & \rightarrow \Lambda^{d} V \\
\left(q,\left(w_{i, j}\right)_{i, j}\right) & \mapsto \sum_{\substack{\alpha_{i, j} \in \mathbb{Z}_{\geq 0} \\
\sum_{i=1}^{d-1} \sum_{j=1}^{n_{i}} i \cdot \alpha_{i, j}=d}} p_{\alpha}(q) \cdot \bigwedge_{i=1}^{d-1} \bigwedge_{j=1}^{n_{i}} \bigwedge_{\ell=1}^{\alpha_{i, j}} w_{i, j}
\end{aligned}
$$

for some polynomial functions $p_{\alpha}: \bigwedge^{d} U \rightarrow K$.

\subsection{Conclusion of the proof.}

Proof of Theorem 9 To bound the strength of elements of $X(V)$ independently of $V$, by the induction assumption applied to $Y$, it suffices to bound the strength of elements of $Z(V)$ independently of $V$. Lemma 25, which focusses the attention to $Z^{\prime}$, and Lemma 26 together reduce this problem further to bounding the strength of elements $q_{d}$ for all $\left(q_{0}, \ldots, q_{d}\right) \in Z^{\prime}(V)$ independently of $V$. Lemma27 shows that such a $q_{d}$ is contained in the image of a covariant. So Lemma 28 implies that the strength of $q_{d}$ is bounded by

$$
\sum_{i=1}^{\lfloor d / 2\rfloor} \operatorname{dim} \bigwedge^{d-i} U
$$

which completes the proof.

Remark 29. It follows from the induction that $N$ from Theorem 9 can be taken equal to

$$
\operatorname{dim} U+\sum_{i=1}^{\lfloor d / 2\rfloor} \operatorname{dim} \bigwedge^{d-i} U
$$




\section{Proof for ordinary tensors}

In this section we adapt the proof from Section 2 to a proof of Theorem 10 for ordinary tensors. Throughout this section, we will let $X$ be a closed subset of $T^{d}$ and $U \in \operatorname{Vec}^{d}$ a tuple of finite-dimensional vector spaces such that $X(U) \subsetneq T^{d} U$.

4.1. Homogeneity. We equip the coordinate ring $K\left[T^{d} V\right]$ with the $d \cdot \mathbb{Z}_{\geq 0}$-grading in which the elements of $\left(T^{d} V\right)^{*}$ have degree $d$. For any closed $X \subseteq T^{d}$ we find, from the fact that $X(V)$ is stable under GL(V), that the ideal $\mathcal{I}(X(V)) \subseteq K\left[T^{d} V\right]$ is stable under $\mathrm{GL}(V)$ and in particular homogeneous. We define

$$
\delta_{X}:=\min _{f \in I(X(U)) \backslash\{0\}} \operatorname{deg} f \in \mathbb{Z}_{\geq 0} .
$$

4.2. Induction. If $\delta_{X}=0$, then we find that $X(V)=\emptyset$ for all $V \in \operatorname{Vec}^{d}$. We may therefore assume that $\delta_{X}>0$ and we proceed by induction, assuming that the theorem holds for all $Y \subseteq T^{d}$ with $Y(U) \subseteq T^{d} U$ and $\delta_{Y}<\delta_{X}$.

4.3. Derivative. Let $f \in \mathcal{I}(X(U)) \backslash\{0\}$ be a homogeneous polynomial of degree $\delta_{X}$. Then, there exists an $r \in T^{d} U$ such that the directional derivative $h:=\frac{\partial f}{\partial r}$ is not the zero polynomial and we may assume that $r=u_{1} \otimes \ldots \otimes u_{d}$ for some $u_{i} \in U_{i}$.

We define the closed subset $Y \subsetneq T^{d}$ by

$$
Y(V):=\left\{q \in X(V) \mid \forall \varphi_{i} \in \operatorname{Hom}_{V e c}\left(V_{i}, U_{i}\right): h\left(\left(T^{d} \varphi\right) q\right)=0\right\}
$$

and note that, by the induction hypothesis, the theorem holds for $Y$. We define

$$
Z(V):=X(V) \backslash Y(V)
$$

and prove that all elements in $Z(V)$ have bounded strength independent of $V$.

4.4. Shifting. For $V \in \operatorname{Vec}^{d}$ we define

$$
\begin{aligned}
& P^{\prime}(V):=T^{d}(U \oplus V)=\bigoplus_{J \subseteq[d]}\left(\bigotimes_{j \in[d] \backslash J} U_{j} \otimes \bigotimes_{j \in J} V_{j}\right), \\
& X^{\prime}(V):=X(U \oplus V) \subseteq P^{\prime}(V), \\
& Z^{\prime}(V):=\left\{q \in X^{\prime}(V) \mid h\left(\left(T^{d}\left(1_{U_{i}} \oplus 0_{V_{i} \rightarrow U_{i}}\right)_{i}\right) q\right) \neq 0\right\} .
\end{aligned}
$$

We think of $P^{\prime}(V), X^{\prime}(V)$ as varieties over $T^{d} U, X(U)$, respectively, via the linear map $T^{d}\left(1_{U_{i}} \oplus 0_{V_{i} \rightarrow U_{i}}\right)_{i}$. We will write $h$ for $h \circ T^{d}\left(1_{U_{i}} \oplus 0_{V_{i} \rightarrow U_{i}}\right)_{i}$.

Lemma 30. We have

$$
Z(U \oplus V)=\bigcup_{g \in \mathrm{GL}(U \oplus V)} g Z^{\prime}(V) .
$$

In particular, $\sup _{q \in Z(U \oplus V)} S(q)=\sup _{q \in Z^{\prime}(V)} S(q)$.

Lemma 31. We have

$$
\sup _{V \in \operatorname{Vec}^{d}} \sup _{q \in X(V)} S(q)=\sup _{V \in \operatorname{Vec}^{d}} \sup \left\{\sup _{q \in Y(V)} S(q), \sup _{q \in Z^{\prime}(V)} S(q)\right\} .
$$


4.5. Chopping. We write $n_{\ell}:=\operatorname{dim} U_{\ell}$ for $\ell=1, \ldots, d$.

Lemma 32. For $q \in P^{\prime}(V)$ write $q=\sum_{J \subseteq[d]} q_{J}$ with $q_{J} \in \bigotimes_{j \in[d] J J} U_{j} \otimes \bigotimes_{j \in J} V_{j}$. Then

$$
S(q) \leq n_{1}+\cdots+n_{d}+S\left(q_{[d]}\right) \text {. }
$$

4.6. Embedding. Define

$$
Q^{\prime}(V):=P^{\prime}(V) / T^{d} V=\bigoplus_{J \subseteq[d]}\left(\bigotimes_{j \in[d] \backslash J} u_{j} \otimes \bigotimes_{j \in J} V_{j}\right)
$$

and write $\pi(V): P^{\prime}(V) \rightarrow Q^{\prime}(V)$ for the natural projection. Let

$$
B(V):=\left\{q \in Q^{\prime}(V) \mid h(q) \neq 0\right\}=\left\{\left(q_{J}\right)_{J \subseteq[d]} \in Q^{\prime}(V) \mid h\left(q_{\emptyset}\right) \neq 0\right\},
$$

an open set in $Q_{U}^{\prime}(V)$. Then $\pi(V)$ maps $Z^{\prime}(V)$ into $B(V)$.

\subsection{An equivariant map back.}

Lemma 33. There exists a GL(V)-equivariant polynomial map

$$
\Psi: Q^{\prime}(V) \rightarrow T^{d} V
$$

such that, for all $q=\left(q_{J}\right)_{J \subseteq[d]} \in Z^{\prime}(V), q_{[d]}$ is a scalar multiple of $\Psi\left(q_{J}\right)_{J \subseteq[d]}$.

Proof. For $x=\left(x_{1}, \ldots, x_{d}\right) \in V_{1}^{*} \times \cdots \times V_{d^{\prime}}^{*}$ let $\varphi_{x, i}: V_{i} \rightarrow U_{i}$ be the linear map $v_{i} \mapsto x_{i}\left(v_{i}\right) u_{i}$; here $u_{1}, \ldots, u_{d}$ are the vectors used in the definition of $h$. Note that $x \mapsto \varphi_{x, i}$ is a $\mathrm{GL}(V)$-equivariant linear map.

For all $t_{1}, \ldots, t_{d} \in K$ define the linear map $\Phi_{x}(t):=T^{d}\left(1_{U_{i}} \oplus t_{i} \varphi_{x, i}\right)_{i}: P^{\prime}(V) \rightarrow T^{d} U$. The restriction of $\Phi_{x}(t)$ to the summand

$$
\bigotimes_{j \in[d] \backslash J} U_{j} \otimes \bigotimes_{j \in J} V_{j}
$$

equals $\prod_{i \in J} t_{i} \cdot \Phi_{x, J}$ where $\Phi_{x, J}$ is the map $\bigotimes_{j \in[d] \backslash J} 1_{u_{j}} \otimes \bigotimes_{j \in J} \varphi_{x, j}$. Note that $x \mapsto \Phi_{x, J}$ is a GL $(V)$-equivariant polynomial map of (ordinary) degree $|J|$ and that $x \mapsto \Phi_{x,[d]}$ is multilinear.

By functoriality of $X$, we have $\Phi_{x}(t)\left(X^{\prime}(V)\right) \subseteq X(U)$, and for $q=\left(q_{J}\right)_{J \subseteq[d]} \in P^{\prime}(V)$ we find that

$$
f\left(\Phi_{x}(t)\left(q_{J}\right)_{J \subseteq[d]}\right)=f\left(\sum_{J \subseteq[d]}\left(\prod_{i \in J} t_{i} \cdot \Phi_{x, J} q_{J}\right)+t_{1} \ldots t_{d} \cdot\left(T^{d} \varphi_{x}\right) q_{[d]}\right),
$$

and this expression vanishes for $q \in X^{\prime}(V)$. The coefficient of $t_{1} \ldots t_{d}$ in the Taylor expansion of this expression equals

$$
h\left(q_{\emptyset}\right)\left(x_{1} \otimes \cdots \otimes x_{d}\right) q_{[d]}+\Psi\left(x,\left(q_{J}\right)_{J \subsetneq[d]}\right)
$$

where the function $\Psi: V_{1}^{*} \times \cdots \times V_{d}^{*} \times Q^{\prime}(V) \rightarrow K$ is $\mathrm{GL}(V)$-invariant and multilinear in $V_{1}^{*} \times \cdots \times V_{d}^{*}$. We note that for $q \in Z^{\prime}(V)$, we have $h\left(q_{\emptyset}\right) \neq 0$ by definition of $Z^{\prime}(V)$, and therefore

$$
\left(x_{1} \otimes \cdots \otimes x_{d}\right) q_{[d]}=-\frac{1}{h\left(q_{\emptyset}\right)} \Psi\left(x,\left(q_{J}\right)_{J \subsetneq[d]}\right) .
$$

The map $\Psi$ factors as the composition of

$$
\begin{aligned}
V_{1}^{*} \times \cdots \times V_{d}^{*} \times Q^{\prime}(V) & \rightarrow\left(V_{1}^{*} \otimes \cdots \otimes V_{d}^{*}\right) \times Q^{\prime}(V) \\
\left(x, q^{\prime}\right) & \mapsto\left(x_{1} \otimes \cdots \otimes x_{d}, q^{\prime}\right)
\end{aligned}
$$


and a unique $\mathrm{GL}(V)$-equivariant map $\left(V_{1}^{*} \otimes \cdots \otimes V_{d}^{*}\right) \times Q^{\prime}(V) \rightarrow K$. We denote the latter map also by $\Psi$, which is now linear in its first argument. If we re-interpret $\Psi$ as a $\mathrm{GL}(V)$-equivariant polynomial map $Q^{\prime}(V) \rightarrow T^{d} V$, then

$$
q_{[d]}=-\frac{1}{h\left(q_{\emptyset}\right)} \Psi\left(q_{J}\right)_{J \subsetneq[d]} .
$$

for all $q \in Z^{\prime}(V)$.

4.8. Covariants. A covariant of $Q^{\prime}(V)$ is a $\mathrm{GL}(V)$-equivariant polynomial map $Q^{\prime}(V) \rightarrow T^{d} V$. So the map $\Psi$ constructed in 4.7 is a covariant. For each nonempty subset $j \subsetneq[d]$, choose a basis $u_{J, 1}, \ldots, u_{J, n_{J}}$ of $\bigotimes_{j \in[d] \backslash J} U_{j}$. Then the map

$$
\begin{aligned}
& \Phi: \bigoplus_{\substack{J \subseteq[d] \\
J \neq \emptyset}}\left(\bigotimes_{j \in J} V_{j}\right)^{\oplus n_{J}} \rightarrow \bigoplus_{\substack{J \subseteq[d] \\
J \neq \emptyset}}\left(\bigotimes_{j \in[d] \backslash J} u_{j} \otimes \bigotimes_{j \in J} V_{j}\right) \\
& \left(w_{J, \ell}\right)_{J, \ell} \mapsto\left(\sum_{\ell=1}^{\prod_{j \in[d] \backslash J} \operatorname{dim} u_{j}} u_{J, \ell} \otimes w_{J, \ell}\right)_{J}
\end{aligned}
$$

is a $\mathrm{GL}(V)$-equivariant isomorphism and the following lemma holds.

Lemma 34. Let $\Psi: Q^{\prime}(V) \rightarrow T^{d} V$ be a covariant. Then the composition $\Psi \circ\left(\operatorname{id}_{T^{d} U}, \Phi\right)$ is of the form

$$
\begin{aligned}
T^{d} U \oplus \bigoplus_{\substack{J \subseteq[d] \\
J \neq \emptyset}}\left(\bigotimes_{j \in J} V_{j}\right)^{\oplus n_{J}} & \rightarrow T^{d} V \\
\left(q,\left(w_{J, \ell}\right)_{J, \ell}\right) & \mapsto \sum_{\left\{J_{1}, \ldots, J_{k}\right\} \in \mathcal{J}} \sum_{\ell_{1} \in\left[n_{I_{1}}\right]} \cdots \sum_{\ell_{k} \in\left[n_{J_{k}}\right]} p_{\left\{J_{1}, \ldots, J_{k}\right\}, \ell_{1}, \ldots, \ell_{k}}(q) \cdot \bigotimes_{i=1}^{k} w_{J_{i}, \ell_{i}}
\end{aligned}
$$

for some polynomial functions

$$
p_{\left\{J_{1}, \ldots, J_{k}\right\}, \ell_{1}, \ldots, \ell_{k}}: T^{d} U \rightarrow K
$$

where $\mathcal{J}$ consists of all unordered partitions of $[d]$ into nonempty sets, i.e., all collections $\left\{J_{1}, \ldots, J_{k}\right\}$ of non-empty subsets $J_{i} \subsetneq[d]$ with $J_{i} \cap J_{i^{\prime}}=\emptyset$ if $i \neq i^{\prime}$ and $\bigcup_{i=1}^{k} J_{i}=[d]$.

\subsection{Conclusion of the proof.}

Proof of Theorem 10 To bound the strength of elements of $X(V)$ independently of $V$, by the induction assumption applied to $Y$, it suffices to bound the strength of elements of $Z(V)$ independently of $V$. Lemma 31 and Lemma 32 together reduce this problem further to bounding the strength of elements $q_{[d]}$ for all $\left(q_{J}\right)_{J \subseteq[d]} \in Z^{\prime}(V)$ independently of $V$. Lemma 33 shows that such a $q_{[d]}$ is contained in the image of a covariant. So using Lemma 34, we see that $q_{[d]}$ is a linear combination of tensor products of elements $w_{J, \ell} \in \bigotimes_{j \in J} V_{j}$ where $J$ ranges over non-empty subsets of $[d]$ and $j$ ranges from 1 to $\prod_{j \in[d] \backslash J} \operatorname{dim} U_{j}$. Fix an integer $m \in[d]$. Then we note for 
each $\left\{J_{1}, \ldots, J_{k}\right\} \in \mathcal{J}$ that $m \in J_{i}$ for some $i \in[k]$. So the strength of $q_{[d]}$ is at most

$$
\begin{aligned}
& \#\left\{w_{J, \ell} \mid m \in J \subsetneq[d], \ell \in\left[\prod_{j \in[d] \backslash J} \operatorname{dim} U_{j}\right]\right\} \\
\leq & \operatorname{dim} U_{m} \cdot \sum_{J \subsetneq([d] \backslash\{m\})} \prod_{j \in J} \operatorname{dim} U_{j} \\
= & \operatorname{dim} U_{m} \cdot\left(\prod_{j \in[d] \backslash\{m\}}\left(\operatorname{dim} U_{j}+1\right)-\prod_{j \in[d] \backslash\{m\}} \operatorname{dim} U_{j}\right) \\
= & \prod_{j \in[d]}\left(\operatorname{dim} U_{j}+1\right)-\prod_{j \in[d] \backslash\{m\}}\left(\operatorname{dim} U_{j}+1\right)-\prod_{j \in[d]} \operatorname{dim} U_{j}
\end{aligned}
$$

and the latter expression is minimized over $m$ when $\prod_{j \in[d] \backslash\{m\}}\left(\operatorname{dim} U_{j}+1\right)$ is maximal, which happens exactly when $\operatorname{dim} U_{m}$ is minimal. This bounds the strength of $q_{[d]}$ independently of $V$.

Remark 35. It follows from the induction that $N$ from Theorem 10 can be taken equal to

$$
n_{1}+\cdots+n_{d}+n_{m} \prod_{j \in[d] \backslash\{m\}}\left(n_{j}+1\right)-\prod_{j \in[d]} n_{j}
$$

where $n_{\ell}=\operatorname{dim} U_{\ell}$ and where $n_{m} \leq n_{\ell}$ for all $\ell \in[d]$.

Remark 36. The definitions of strength we have used have the following common generalisation: For integers $0 \leq m \leq n, d_{1}, \ldots, d_{n} \in \mathbb{N}$ with $\sum_{i} d_{i} \geq 2$, and vector spaces $V_{1}, \ldots, V_{n} \in \mathrm{Vec}$, the strength $S(q)$ of a composite tensor

$$
q \in S^{d_{1}} V_{1} \otimes \cdots \otimes S^{d_{m}} V_{m} \otimes \bigwedge^{d_{m+1}} V_{m+1} \otimes \cdots \otimes \bigwedge^{d_{n}} V_{n}
$$

is the minimal number $k$ of terms in any composition of the form

$$
q=r_{1} s_{1}+\cdots+r_{k} s_{k}
$$

where

$$
\begin{aligned}
& r_{i} \in \quad S^{e_{1}} V_{1} \otimes \cdots \otimes S^{e_{m}} V_{m} \otimes \bigwedge^{e_{m+1}} V_{m+1} \otimes \cdots \otimes \bigwedge^{e_{n}} V_{n} \\
& s_{i} \in S^{d_{1}-e_{1}} V_{1} \otimes \cdots \otimes S^{d_{m}-e_{m}} V_{m} \otimes \bigwedge^{d_{m+1}-e_{m+1}} V_{m+1} \otimes \cdots \otimes \bigwedge^{d_{n}-e_{n}} V_{n}
\end{aligned}
$$

for suitable $0 \leq e_{i} \leq d_{i}$ with $\left(e_{1}, \ldots, e_{n}\right) \neq(0, \ldots, 0),\left(d_{1}, \ldots, d_{n}\right)$.

A version of the main theorem for composite tensors generalising the three versions exists. A proof of this version can be obtained by modifying the proof in this section. The most important changes are:

(a) we must assume that $\operatorname{char} K=0$ or $\operatorname{char} K>d_{i}$ for all $i \in\{1, \ldots, n\}$;

(b) we take $h:=\frac{\partial f}{\partial r}$ where $r=r_{1} \otimes \cdots \otimes r_{n}$ with $r_{i}=u_{i}^{d_{i}}, u_{i} \in U_{i}$ for $i \leq m$ and $r_{i}=u_{i, 1} \wedge \cdots \wedge u_{i, d_{i}}, u_{i, j} \in U_{i}$ for $i>m$; and

(c) for $i \leq m$ we take $x_{i} \in V_{i}^{*}$ and $t_{i} \in K$, for $i>m$ we take $x_{i, j} \in V_{i}^{*}$ and $t_{i, j} \in K$ and we let $\Phi_{x}(t)=T^{d}\left(\Phi_{x_{1}}^{(1)}\left(t_{1}\right), \ldots, \Phi_{x_{n}}^{(n)}\left(t_{n}\right)\right)$ where $\Phi_{x_{i}}^{(i)}\left(t_{i}\right)$ is the map from the symmetric case for $i \leq m$ and the map from the alternating case for $i>m$.

In addition, the bounds must be adjusted to (more complicated) expressions. 


\section{Proof over $\mathbb{Z}$.}

Proof of Theorems 12, 14 and 15 For $P \in\left\{S^{d}, \wedge^{d}, T^{d}\right\}$ we write $P_{\mathbb{Z}}$ for the version over $\mathbb{Z}$ and $P_{K}$ for the version over $K$. As in the proofs over $K$, let $U$ be a $\mathbb{Z}$-module for which $X_{\mathbb{Z}}(U) \neq P_{\mathbb{Z}} U$, and let $f$ be a nonzero, homogeneous polynomial with integral coefficients vanishing on $X_{\mathbb{Z}}(U)$. For any field $K$ whose characteristic does not divide all coefficients of $f$, note that $f$ specialises to a nonzero polynomial vanishing on $X_{K}\left(U_{K}\right)$. As the specialisation of $f$ is defined over the prime field, it follows that if $p=\operatorname{char} K>0$ and all directional derivatives of $f$ are zero over $K$, then $f$ is a $p$-th power, and one can replace it by its $p$-th root. We let $h$ be the partial derivative of $f$ in the direction of a vector in such a way such that $h$ and $\Psi$ are also defined over the prime field. Now $\Psi$ has the required properties by Remark 11 since it has those properties over any infinite field $L \supseteq K$. It follows that the bound from Remark 23, 29 or 35 applies. Note that this deals with all but finitely many characteristics. For each of the remaining characteristics $p$, if $K$ is a field of characteristic $p$ such that $X_{K} \subsetneq P_{K}$, then there exists a $U \in \operatorname{Vec}_{\mathbb{Z}}$ and an $f$ as above that specialises to a nonzero polynomial vanishing on $X_{K}\left(U_{K}\right)$. So we can take for $N$ the maximum of finitely many numbers of the form of Remark 23, 29, 35

\section{REFERENCES}

[AH16] Tigran Ananyan and Melvin Hochster. Small subalgebras of polynomial rings and Stillman's conjecture. 2016. Preprint, arXiv:1610.09268

[Chi02] Jaydeep V. Chipalkatti. Decomposable ternary cubics. Exp. Math., 11(1):69-80, 2002.

[DES17] Harm Derksen, Rob Eggermont, and Andrew Snowden. Topological noetherianity for cubic polynomials. Algebra Number Theory, 11(9):2197-2212, 2017.

[Dra17] Jan Draisma. Topological Noetherianity of polynomial functors. 2017. Preprint, submitted, arXiv:1705.01419

[KZ18] David Kazhdan and Tamar Ziegler. On ranks of polynomials. 2018. Preprint, arXiv:1802.04984

[Lan12] J. M. Landsberg. Tensors: geometry and applications, volume 128 of Graduate Studies in Mathematics. American Mathematical Society (AMS), Providence, RI, 2012.

[TS16] Terence Tao and Will Sawin. Notes on the "slice rank" of tensors. 2016. https://terrytao. wordpress. com/2016/08/24/notes-on-the-slice-rank-of-tensors/.

Universität Bern, Mathematisches Institut, Alpeneggstrasse 22, 3012 Bern, Switzerland

E-mail address: arthur.bik@math.unibe.ch

Universität Bern, Mathematisches Institut, Sidlerstrasse 5, 3012 Bern, Switzerland; and EindHOVEN UNIVERSITY OF TECHNOLOGY

E-mail address: jan.draisma@math.unibe.ch

Eindhoven University of Technology, Department of Mathematics and Computer Science, P.O. Box 513, 5600 MB Eindhoven, The Netherlands

E-mail address: r.h.eggermont@tue.nl 